\title{
Terminologie vernaculaire du chant et discours de spécialité en Basse-Bretagne (XIX ${ }^{\mathrm{e}}-\mathrm{XX}^{\mathrm{e}}$ siècles)
}

19th and 20th Century vernacular song and specialised field discourse

terminology (Western Brittany, France)

\section{Hervé Rivière}

\section{OpenEdition}

\section{Journals}

Édition électronique

URL : http://journals.openedition.org/ethnomusicologie/1569

ISSN : 2235-7688

\section{Éditeur}

ADEM - Ateliers d'ethnomusicologie

\section{Édition imprimée}

Date de publication : 1 janvier 1998

Pagination : $57-71$

ISBN : 2-8257-0639-6

ISSN : 1662-372X

\section{Référence électronique}

Hervé Rivière, «Terminologie vernaculaire du chant et discours de spécialité en Basse-Bretagne (XIXeXXe siècles) », Cahiers d'ethnomusicologie [En ligne], 11 | 1998, mis en ligne le 07 janvier 2012, consulté le 03 mai 2019. URL : http://journals.openedition.org/ethnomusicologie/1569

Ce document a été généré automatiquement le 3 mai 2019.

Tous droits réservés 


\section{Terminologie vernaculaire du chant et discours de spécialité en Basse-Bretagne (XIX ${ }^{e}-X X^{e}$ siècles)}

19th and 20th Century vernacular song and specialised field discourse

terminology (Western Brittany, France)

\section{Hervé Rivière}

1 L'ethnomusicologue qui s'intéresse au patrimoine chanté de Basse-Bretagne - c'est-à-dire la partie celtophone de la péninsule armoricaine - dispose d'un travail de catégorisation déjà effectué par les folkloristes et les ethnologues bretons depuis plus d'un siècle. L'homogénéité de cette littérature sur les "genres» poétiques et musicaux est remarquable. C'est comme si le discours de spécialité s'était figé autour de catégories définies une fois pour toutes et reproduites depuis lors à l'infini. La classification des folkloristes étant fondée sur celle de détenteurs de la tradition chantée, sa légitimité va $a$ priori de soi. Ce qui entre sans doute pour beaucoup dans les raisons de sa pérennité. Mais la pertinence culturelle de cette classification élaborée au début du XIXe siècle a-t-elle été vérifiée par la suite sur l'ensemble des aires de collectes? Il semble bien que non, comme il semble bien y avoir, malgré la prétendue référence autochtone, une distorsion parfois sensible entre cette dernière et le discours savant.

L'exemple le plus flagrant de cette distorsion est peut-être celui des « cantiques », qui «se trouvent dans une situation difficile à définir " (Quellien 1899: 16). Dans les pages qui suivent, nous prendrons comme prétexte ce cas des chants religieux pour réexaminer quelques unes des données à prendre en compte pour une catégorisation de la poésie chantée en langue bretonne. 
3 Tentons, pour nous-même, une première définition. Un « cantique » est sinon composé, du moins avalisé par l'autorité ecclésiale. Il est proféré soit directement sous le contrôle de cette dernière - dans un lieu saint ou sacralisé (église, chapelle, parcours processionnel, etc.) -, soit indirectement (intimité domestique pieuse, par exemple) ${ }^{1}$. Si son titre ne contient pas de terme spécifique le désignant, la présence, dans l'intitulé, d'adjectifs comme «spirituel », « santel », " devot » ( "spirituel », " saint », " dévot »), etc. ou une référence explicite à un saint peut en constituer une marque d'identification (par exemple : « Gwerz Santez Barba e Rosco », « Chant de Sainte Barbe à Roscoff »).

4 L'épithète «spirituel » renvoie explicitement à celui des dénominations «cantique spirituel » et «chanson spirituelle » largement répandues en France à partir du Concile de Trente (1545-1563) (cf. par exemple les Chansons spirituelles sur la Sainte Cène publiées à Genève en 1546, les Chansons spirituelles a l'honneur \& louange de Dieu [...] de Muret et Goudimel publiées en 1555, ou encore le Beau recueil de plusieurs belles chansons spirituelles, avec ceux des huguenots heretiques \& ennemis de Dieu, \& de notre mere saincte Eglise, et Nouvelles chansons spirituelles, pour recreer les esprits des Catholiques, afliges des ennemis, \& adversaires de la Foy, œuvre conjointe de Christofle de Bourdeaux et de F. Legier Bontemps publiée à Paris après 1569).

5 Quant à l'acception de "cantique » dans un sens autre que celui de la référence aux poèmes bibliques, elle est semble-t-il attribuable à Nicolas Denisot (né en 1515) (Gastoué 1924 : 157-159). «Cantique » désigne alors « une forme poétique aux strophes régulières [...] (Gastoué 1924: 158), et ne concerne que le texte (Gastoué 1924: 158, et Hameline 1981 : 251). Ainsi, Claude Hopil, qui publie respectivement en 1604 et 1633 des « cantiques spirituels » et des « chansons spirituelles » dissocie clairement les poésies destinées à être chantées de celles qui ont été écrites sans souci de les mettre en musique. Cependant, «cantique» et «chanson spirituelle» - ainsi que l'expression croisée «cantique spirituel» (cf. par exemple les Cantiques spirituels de Mathurin Cordier publiés en 1560 ou les Paraphrases des hymnes et cantiques spirituels [...] de Michel Coissard publiés en 1592) ne tarderont pas à s'employer l'un pour l'autre (Hameline 1981 : 251).

6 A cette époque, le terme «cantique » s'applique en fait aussi bien au répertoire profane que religieux, même si la tendance est de le réserver à ce dernier domaine (Gastoué 1924 : 158-159). Il gardera longtemps son sens de simple poésie (Launay 1993: 51), ce qui explique la présence de la mention kantik sur les feuilles volantes de colportage du XVIIIe siècle en Bretagne pour désigner un chant profane : Cantic nevoe voar sujet pevarzec den a so bet beuet er memes bag o vont d'ar goemon ("Cantique nouveau au sujet de quatorze personnes qui se sont noyées dans le même bateau, en allant au goémon ») (Giraudon 1985 : 11 et 16). Cela dit, le sens de «kantik» sur ces feuilles volantes n'est pas - ou du moins n'est plus - le sens commun du breton parlé contemporain.

7 Toutes ces dénominations françaises seront reprises en breton, selon des traductions diverses qui se maintiendront dans la langue jusqu'au $\mathrm{XX}^{\mathrm{e}}$ siècle. En témoignent notamment les Canticou spirituel da beza canet er catechismou... («Cantiques spirituels à chanter au catéchisme...») publiés en 1642, les Cantiqueu spirituel ar deverieu ar christen ("Cantiques spirituels sur les devoirs du chrétien») de Pierre Barisy (1710), les Gucrzonneu santél («Chansons spirituelles») publiées en 1734, les Canticou spirituel composet evit usaich ar Missionou («Cantiques spirituels composés pour l'usage des Missions ») de Guillaume Le Jeune (1784), le Choés a gannenneu spirituel aveit er Retraid («Choix de chansons spirituelles pour la retraite ») publié en 1792, les Canticou nevez evit 
usach ar missionou, ar retrejou hac ar c'hatemismou ("Nouveaux cantiques pour l'usage des missions, des retraites et des catéchismes») publiés en 1833, les Kanaouennou santel dilennet ha reizet evit Eskopti Kemper («Chansons spirituelles choisies et mises en ordre pour l'Évêché de Quimper ») de Guillaume Henry (publiées en 1842), etc.

Examinons les termes figurant sur les feuilles volantes ronéotypées ou photocopiées distribuées aux fidèles les jours de pardon (fête locale), sur les livrets paroissiaux et les opuscules divers en usage actuellement dans les églises de Basse-Bretagne ${ }^{2}$. Apparaissent principalement : kantik, kanenn, gwerz, gwerzenn, pedenn et buhez. Les deux derniers termes ("prière» et «vie») ne renvoyant pas d'emblée, dans la langue commune, à une production versifiée et chantée, nous les laisserons de côté. Reste kantik, kanenn, gwerz et gwerzenn dont la répartition géographique suscite plusieurs observations ( $c f$. cartes 1 et 2). Tout d'abord, on remarque des distributions complémentaires, d'une part, entre kantik et kanenn, et, d'autre part, entre gwerz et gwerzenn. Ensuite, on note une distribution sensiblement équivalente, d'une part entre kantik et gwerz, et, d'autre part, entre kanenn et gwerzenn. Enfin, les aires géographiques ainsi définies se superposent quasiment trait pour trait aux aires dialectales KLT et vannetaise ${ }^{3}$.

Carte 1 : aires de répartition des deux principaux termes désignant un cantique

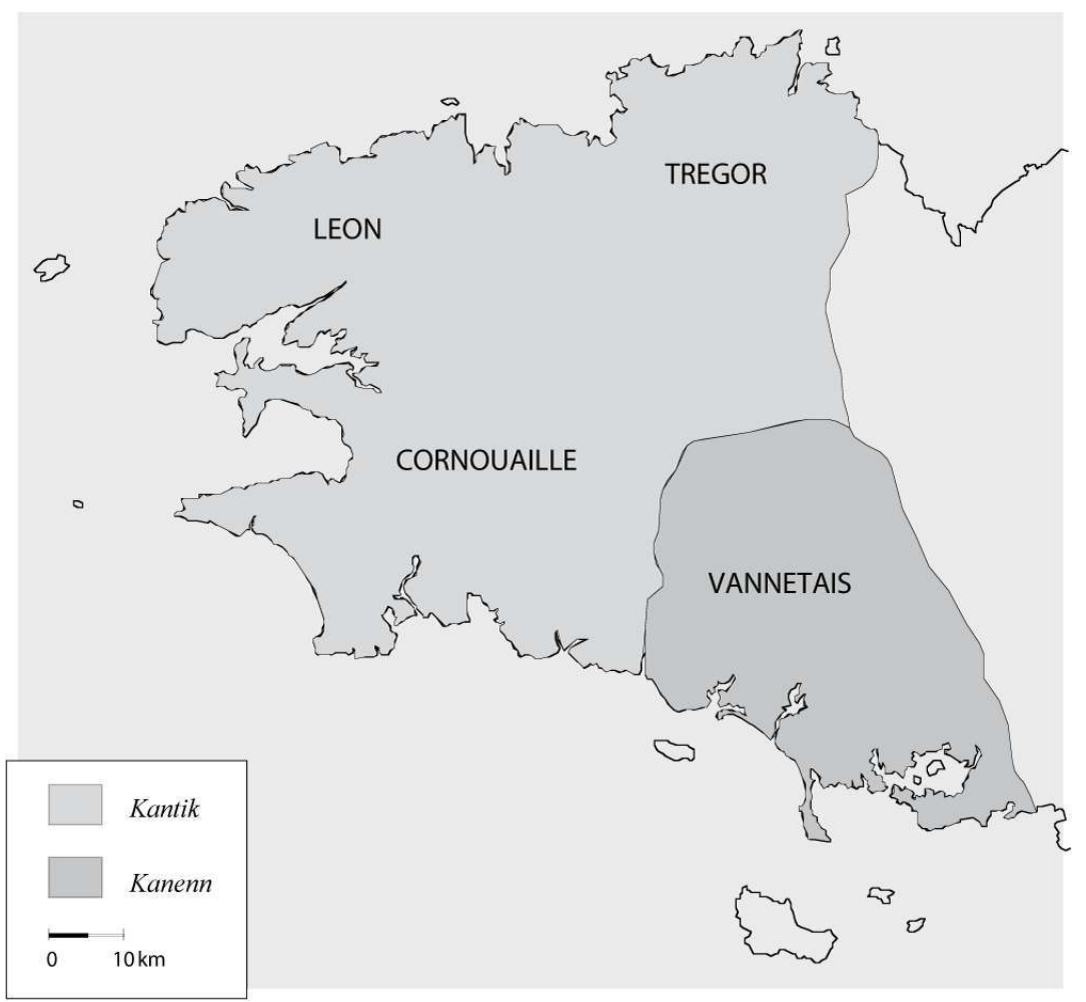

Le lexème prépondérant KLT kantik, emprunté au français - si ce n'est un emprunt direct au latin canticum -, désigne de manière monovalente un «cantique » - tel que nous l'avons défini plus haut. Il trouve son équivalent haut-vannetais dans kanenn. En revanche, ce dernier terme, dans son acception bas-vannetaise, désigne indifféremment un chant d'Église ou un chant profane. 
10 Kanenn s'inscrit dans une opposition kanenn / son(enn) dont la compréhension semble être la suivante: là - c'est-à-dire en bas-vannetais ${ }^{4}$ - où kanenn indique un "chant " (indifférencié) (cf. carte 3), son désigne une pièce de musique instrumentale ; là - c'est-àdire en haut-vannetais ${ }^{5}$ - où kanenn indique un "chant religieux", sonenn dénote un « chant profane ». Il en va de même pour les verbes auxquels ils renvoient : là où kaniñ ( kanal, kano) signifie de manière générique "chanter ", soniñ (son) signifie "jouer d'un instrument de musique "; là où kaniñ signifie « chanter un chant religieux ", soniñ signifie [ «jouer d'un instrument de musique » - comme précédemment -, mais aussi] «chanter un chant profane» (avec parfois la précision «soniñ g'ar beg», «chanter avec la bouche»).

11 Les termes KLT gwerz et vannetais gwerzenn sont étymologiquement issus de la même racine latine versus ${ }^{6}$. Le second, lorsqu'il renvoie au chant, ne désigne habituellement pas autre chose qu'une hymne religieuse ${ }^{7}$; c'est un synonyme du KLT « kantik» et du hautvannetais « kanenn ». En revanche, le sens du premier est ambigu puisqu'il ne présuppose pas d'un contenu profane ou religieux.

"[Les gwerziou] vont des histoires de saints locaux aux récits fantastiques, historiques et aux faits-divers... » (Laurent $1988: 112)$;

"Complainte édifiante, dramatique, historique, fantastique ou religieuse " (Kemener 1996: 81).

12 Donatien Laurent rappelle, dans son étude sur "La gwerz de Skolan...", toute l'importance du rôle joué par « cette vérité à deux faces - vérité d'expérience et vérité des sentiments - qui est le principe vital de la gwerz » (Laurent 1971: 29). La croyance dans l'événement relaté tient tant que ce dernier reste dans le monde des possibles. Ce qui importe, c'est l'acceptabilité d'une trame narrative qui doit atteindre l'expérience humaine de l'auditeur plus que la réalité historique précise (bien que celle-ci se conserve parfois de façon remarquablement fidèle à travers les siècles ${ }^{8}$ ).

«Les gens disent leur peine dans la chanson. Une gwerz peut être encore composée sur des gens qui ont vu du vilain, qui ont été tués, etc. Après qu'ils ont été tués, on compose une chanson sur leur vie [...]. Une gwerz est une chanson triste, une chanson pénible qui dit la vie de quelqu'un, ou la vie qu'il a eue, et qui raconte quelque chose de triste [...]. Mais une vie en tout cas »'.

"Ça peut arriver aux gens. C'est arrivé dans un temps, on n'a pas été vérifier, mais ça peut aussi nous arriver. Importance de la parole, notion de respect par rapport à celui qui a été témoin et qui a transmis dans sa chanson, et qui offre son témoignage [...]. La chanson est plus forte que la parole parlée ou que le conte. Un conte n'est pas vraiment vrai ; on commence par mettre en doute : ur wech e oe... ${ }^{10}$ Un chanteur ne peut tromper un auditoire. Un mot est juste par sa contenance, sa musique. Je ne dirai pas : " une gwerz, c'est un chant de vérité ». C'est trop figer les choses. Il y a de l'irréel, qui est vrai quelque part $»^{11}$.

13 Ainsi le récit se perpétue-t-il, se sédimente-t-il dans le patrimoine oral, selon un schéma tout droit hérité du Moyen âge :

"A nos yeux d'hommes du $\mathrm{XX}^{\mathrm{e}}$ siècle, le grand nombre des intermédiaires fournirait plutôt des raisons de douter de la totale authenticité des faits. A chaque étape de la transmission, une part du vrai se perd et la fable s'enfle. Pour les gens $\mathrm{du} \mathrm{XIV}^{\mathrm{e}}$ siècle, c'était très certainement l'inverse [...]. Plus les garants étaient nombreux, plus l'histoire avait de chances d'être considérée comme véridique " (Martin 1988 : 492). 
14 Un cantique, dès lors qu'il relate la vie - et la mort - d'un saint, possède une trame narrative et est de fait - là où le mot est utilisé - une gwerz à part entière. Les dizaines de cantiques sanctoraux dont les feuilles distribuées lors des pardons ont pour titres « Gwerz sant X » («Gwerz de Saint X ») sont là pour en témoigner («Gwerz Sant Tugdual », « Gwerz de Saint Tugdual », à Landudal ; «Guerz Zant Vaude », " Gwerz de Saint Maudé », à Duault, etc.). Par contre, un chant de piété non narratif (adoration, vénération, louanges, etc.) ne recevra jamais une telle appellation.

Carte 2 : relevé de " gwerz » et de " gwerzenn » dans l'acception de " cantique " (collection de feuilles volantes, Rivière 1994) et de " gwerz » dans celle de « chanson » (d'après Le Roux 1924, fasc. IV, carte 306)

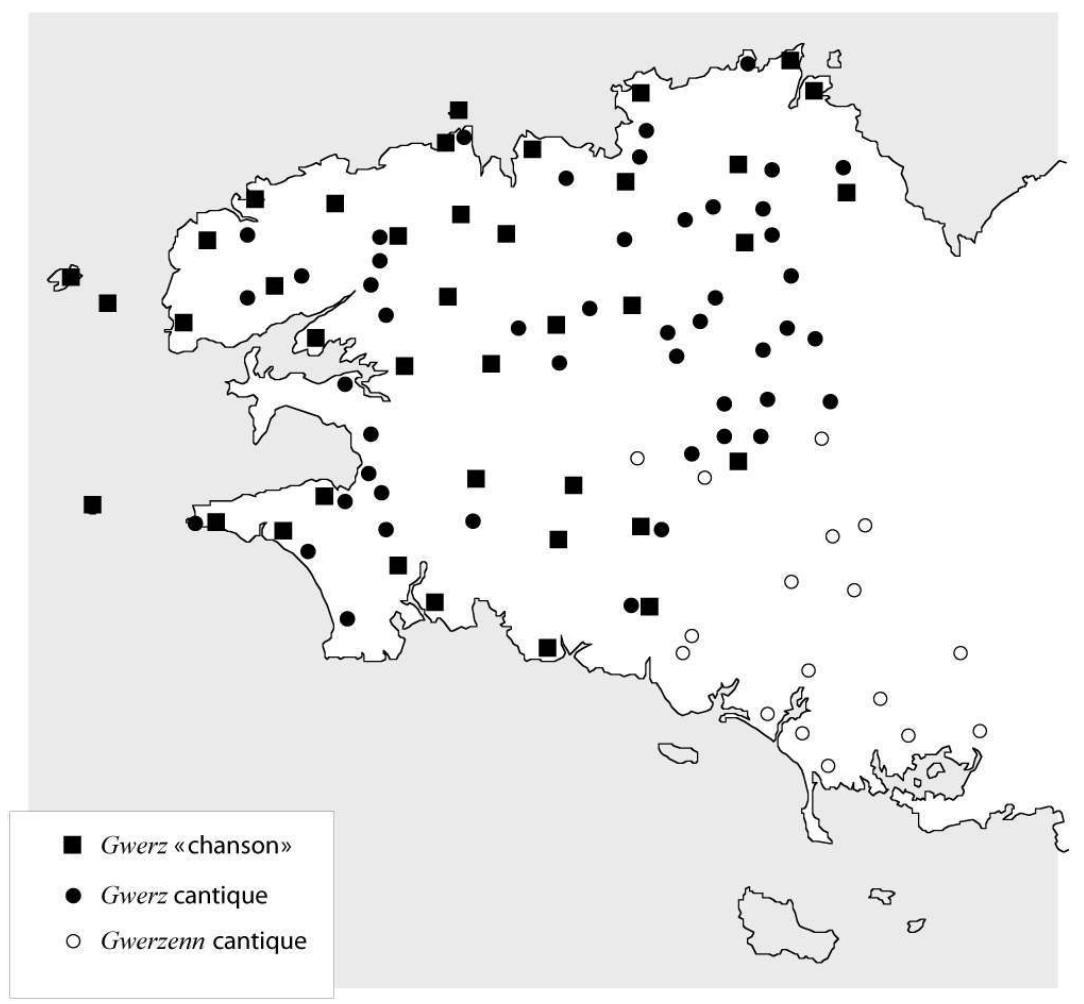

Retenons donc que «gwerz » et «kantik» ne sont pas opposables, car ils s'inscrivent sur deux axes de pertinence peuvant se recouper. Le premier de ces axes est celui de l'appartenance à la catégorie des chants narratifs ; le second celui de l'appartenance à la catégorie des chants d'Église. Ainsi, au gré de ses ré-éditions, un même cantique sanctoral de pardon (chant narratif d'Église) pourra-t-il recevoir l'appellation de «kantik» ou de « gwerz $»^{12}$.

Or, dans le discours de maints spécialistes de littérature orale depuis plus d'un siècle, le positionnement de « gwerz « et de « kantik» l'un par rapport à l'autre se fait sur un même plan classificatoire, chacun des termes désignant une catégorie excluant l'autre - comme nous allons le voir ci-dessous. Ce qui nous paraît non viable au regard de ce que nous venons d'exposer. 
Les folkloristes, les ethnonologues et les musicologues ont pris l'habitude de répartir les chants en langue bretonne selon trois groupes : gwerz, son ${ }^{13}$ et kantik.

C'est au début du XIX ${ }^{\mathrm{e}}$ siècle, sous la plume d'Hersart de La Villemarqué (1815-1895), qu'apparaît cette classification tripartite ${ }^{14}$. Détail important, l'auteur prend soin de préciser que celle-ci n'émane pas d'un travail classificatoire personnel mais du discours de ses informateurs :

« Le classement que j'ai adopté pour les textes n'est autre que celui des chanteurs eux-mêmes : ils ne connaissent plus guère que trois espèces de cantilènes: des chants mythologiques, héroïques, historiques, et des ballades, qu'ils appellent généralement du nom de gwers, et dont ils qualifiaient autrefois quelques-uns de lais; des chants de fête et d'amour qu'ils nomment quelquefois kentel et le plus souvent sôn ou zôn; enfin des légendes et des chants religieux » (1959: 6).

Cette classification sera reprise quelques années plus tard par François-Marie Luzel (1821-1895), qui en profitera pour affiner la teneur des catégories ${ }^{15}$ :

«J'ai divisé ma publication en deux parties, les Gwerziou et les Soniou. Cette division était naturellement indiquée ; elle renferme, à l'exception des cantiques, tout ce qui se chante en breton dans nos campagnes armoricaines. Les Gwerziou comprennent les chansons épiques, qui peuvent se subdiviser en: chansons historiques, chansons légendaires, chansons merveilleuses ou fantastiques, et chansons anecdotiques. - Les Soniou, c'est la poésie lyrique. On comprend sous cette dénomination : les chansons d'amour, les chansons de Kloers ou clercs, qui tiennent une si large place dans la poésie bretonne, - les chansons satiriques et comiques, les chansons de noces et de coutumes, etc. - Il faut y ajouter les chansons d'enfants, les chansons de danse, rondes, jabadaos, passe-pieds, etc. » (1868-1:V).

Luzel précise encore :

"J'en ai fini avec les Gwerziou, ou chants sombres, tragiques, racontant des apparitions surnaturelles, des assassinats, des infanticides, des duels à mort, des trahisons, des enlèvements et des violences de toute sorte [...].

J'arrive, à présent, aux Soniou, où respire un autre ordre d'idées et de sentiments, plus tendres et plus humains: chants d'amour, douces élégies, illusions et désillusions, refrains de danse, jeux et rondes enfantines, etc. » (1874-2 : VI).

1 L'introduction de Chants et chansons populaires de la Bretagne fera autorité et deviendra une référence quasi obligée dans les écrits des spécialistes de littérature orale qui se succèderont jusqu'à nos jours. Elle en viendra même à faire oublier que le véritable père de la tripartition est La Villemarqué. Narcisse Quellien, par exemple (1899:12n), attribue à Luzel l'origine de la catégorie des cantiques, alors que celle-ci est déjà envisagée dans la préface du Barzaz Breiz :

«M. Luzel, outre les gwerziou (chansons épiques ou anecdotiques) et les soniou (poésies lyriques et élégiaques), admet une catégorie de cantiques ou chants religieux. »

Les catégories des folkloristes font largement appel à la thématique littéraire des chants, un critère aux contours parfois difficiles à cerner :

«Le classement d'une chanson dans telle ou telle rubrique donne lieu parfois à flottement et à confusion » (Giraudon : 62).

«Une certaine confusion existe parfois [...] entre gwerzioù et sonioù. C'est le cas, par exemple, pour Iann ann Arc'hantek [...] (Piriou 1989 : 233).

Certains auteurs prennent timidement leurs distances - «Selon la classification de Luzel, [son] s'appliquerait plutôt à une chanson d'inspiration lyrique ou satirique » (Kemener 1996 : 82) -, d'autres en viennent carrément à renoncer à toute définition trop précise, ou 
par se contenter d'opposer vaguement les chants : «On range [dans les Sonioù] tous les chants qui ne sont pas des Gwerzioù ou des Kantikoù » (Le Menn, s.d. : 1). (1924, 2/1977) laisse apparaître des aires bien différenciées d'emploi de gwerz et de son comme traductions du français « chanson ». Certes, une telle enquête laisse perplexe tant elle est fondée sur le présupposé qu'il existe en breton (et dans toutes ses variétés dialectales) un équivalent à "chanson " (pris dans quel sens, d'ailleurs ?). Quoi qu'il en soit, le résultat de cette induction culturelle tend à montrer que les deux termes s'opposeraient d'un point de vue aréal et non d'un point de vue des contenus littéraires, contrairement à ce qu'écrivent les folkloristes ${ }^{16}$. Nous voici donc partagés entre, d'une part, une classification dont le flou relatif est relevé par ceux-là mêmes qui la véhiculent, et, d'autre part, une étude de terrain qui contrarie plus franchement encore cette classification.

Carte 3 : « chanson », d'après Le Roux 1924, fasc. IV, carte 306

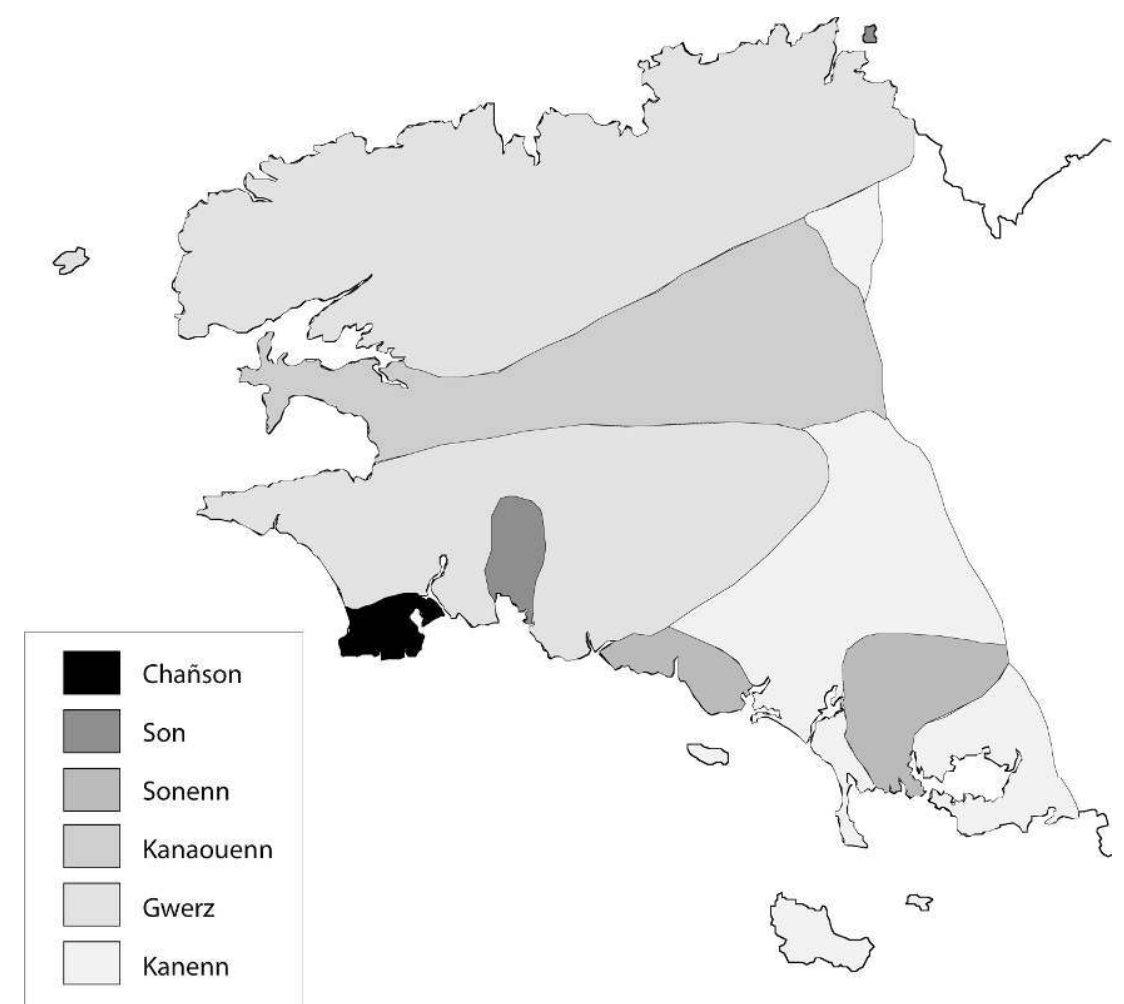

Au vu de la diversité du lexique breton relatif au chant - diversité consignée aussi bien dans l'Atlas de Le Roux que dans les écrits des folkloristes, des littérateurs et des lexicographes ${ }^{17}-$, on peut comprendre la légitimité d'une réduction de la terminologie afin d'unifier un langage de spécialité. Mais les classes façonnées par les folkloristes parviennent-elles à récupérer tous les termes vernaculaires, ou, si l'on préfère, tous les termes recueillis ici et là trouvent-ils leur place dans l'une ou l'autre des catégories? Les chants qualifiés de kentel, par exemple, ne transcendent-ils pas les trois catégories établies? Ouvrons une petite parenthèse historique. «Kentel » est issu du gaulois cant(a)

Cahiers d'ethnomusicologie, 11 | 2011 
$l^{\prime 18}{ }^{18}$, terme qui s'est également maintenu en irlandais et en gallois modernes (respectivement cetal et cathl). Une concurrence entre le latin versus et le celtique cantalon dans les langues brittoniques aboutit à un résultat différent de part et d'autre de la Manche. En Bretagne insulaire, cantalon prit l'acception de "poésie chantée » (gallois cathl) et versus celle de "leçon » (gallois gwers). En Armorique, cantalon en vint à désigner la «leçon" (breton kentel), et versus à s'arroger le domaine de la "poésie chantée » (breton gwerz) ${ }^{19}$.

Nous avons vu plus haut que La Villemarqué donnait aussi, pour la Bretagne armoricaine, kentel comme synonyme de son (Barzaz-Breiz, préface, p. VI) ${ }^{20}$. Peut-être s'agit-il là d'une de ces constructions dont il était friand (cf. Tanguy 1977: passim). Nous n'avons, pour notre part, jamais relevé un tel sens en breton. Et si certains auteurs inscrivent aujourd'hui kentel dans le giron de la poésie chantée, c'est sans doute plus par référence à la préface du Barzaz-Breiz que comme le résultat d'enquêtes de terrain ${ }^{21}$. A moins que cela ne soit par référence à certains titres ou paroles de chansons sur feuilles volantes comprenant kentel, chansons qu' «il faut comprendre [...] comme traitant un sujet [...] prétexte à une leçon de morale (Giraudon 1985: 64)»; en ce cas, le sens de kentel demeurerait quand même celui, premier, de «leçon »).

Sur un autre plan, l'opposition gwerz / son (ou de type gwerz / son) ne peut évidemment se concevoir que là où les deux termes de l'opposition (où leurs équivalents) sont bien représentés. Or,

"On ne trouve pas, en "Bro-Guéned», ces poignantes histoires de rapts, d'envoûtements, d'apparitions magiques et de violences qui sont familières aux populations de la côte trégorroise [...]. Le triomphe des Vannetais, c'est la sône, c'est-à-dire l'élégie, l'idylle, ce que Luzel appelle fort justement «la poésie des femmes, des amoureux et des rêveurs » » (Herrieu et Duhamel 1911 : III-IV).

«Si quelque sombre gwerz, généralement, importée, se chante en ce pays, c'est presque toujours amputée des développements tragiques où les Bretons du Nord se complaisent » (Herrieu et Duhamel 1911 : III).

Yves Le Diberder, bien que contestant une partie de ces propos ${ }^{22}$, les confirme qualitativement :

«Si la poésie populaire du Bro-Erec est plus variée qu'on ne l'admet communément, il est bien certain [...] qu'elle est surtout supérieure dans la chanson lyrique et la chanson poétique » (1911-1912: 534).

Le vannetais ne connaît pas d'opposition lexicale de type gwerz / son. Mais le cas de ce dialecte n'est qu'un exemple ; la diversité des contenus littéraires, des formes poétiques, de la matière musicale, etc. sont d'une telle hétérogénéité en Bretagne qu'il est bien difficile d'imaginer une conception autochtone unique du patrimoine chanté. Ce qui n'empêche pas Quellien d'affirmer :

«Toutes les manifestations de la poésie bretonne sont enfermées sous cette double

forme du gwerz et du sonn» (1899: 14-15).

"On rencontre dans les livres d'autres désignations ; mais elles n'existent pas dans

la langue vulgaire, et le peuple les ignore » $(1899: 12)$.

31 A une époque où les études de littérature orale cherchaient à nommer dans les mêmes termes ce qui était identique - «La question des genres dans la poésie populaire est controversée. Ampère enseigne une division; Bujeaud en adopte une autre; chacun apporte la sienne, s'appuyant sur des raisons qui ont l'air toutes plausibles » (Quellien : 12 n) -, la tripartition - son noyau dur surtout (c'est-à-dire l'opposition gwerz/son) - pouvait donner l'illusion d'une homogénéité de la pensée autochtone bretonne. Moule 
idéologique rassurant d'une spécificité et d'une cohérence conceptuelles d'un patrimoine littéraire vis-à-vis de son voisin roman ou francophone, l'illusion a pu être entretenue jusqu'à nos jours.

Les folkloristes, bien qu'ayant relevé d'une façon ou d'une autre la notion de récit comme critère définitoire d'une gwerz, ont surtout glosé sur la thématique littéraire de cette dernière, une thématique qu'ils ont tenté de caractériser avant tout à partir des chants profanes de tradition purement orale - la littérature « semi-populaire », celle des feuilles volantes et des cantiques, ne les intéressant que dans une moindre mesure. Cette orientation étant prise, le critère formel d'identification se volatilise quelque peu, et, lorsque se présente une problématique comme celle d'une opposition profane / religieux des chants, cette dernière apparaît communément sous la forme son, gwerz / kantik.

Certains auteurs reconnaissent malgré tout des gwerz au contenu religieux, tandis qu'indubitablement, « kantik» est connoté «musique d'Église ». Il y a là une imprécision sur l'opposition profane / religieux, et maints auteurs en viennent à reconnaitre l'osmose entre la catégorie des gwerz et celle des kantik (cf. par exemple Le Menn, s.d. : $1:$ «Il est parfois difficile de faire la distinction entre kantik et gwerz, en particulier lorsque le chant raconte l'histoire - plus ou moins légendaire - des saints »). Mais peut-on conserver le cadre tripartite, qui devient alors incohérent?

L'incohérence est en revanche levée chez ceux qui s'intéressent avant tout aux chants profanes de tradition purement orale. Évacuant la littérature «semi-populaire » des clercs, leur cadre classificatoire se résume à la seule bipartition gwerz/son. En conséquence, ces folkloristes et ethnologues peuvent intégrer explicitement un possible contenu religieux dans la définition de la gwerz (du domaine oral). Reste alors à s'interroger sur les contours d'une catégorie de « cantiques » en tant que tels ${ }^{23}$.

Il est important, nous semble-t-il, d'insister sur l'idée que non seulement une gwerz peut avoir un contenu religieux, mais qu'elle peut également être un cantique officiel - avec " gwerz » pour intitulé. Ne pas le faire, c'est accepter d'être contredit par les sources orales et écrites autochtones.

Mais par-delà la question des catégories profanes et religieuses, ne faudrait-il pas tout simplement remettre à plat l'ensemble des données classificatoires héritées du XIX ${ }^{\mathrm{e}}$ siècle, faire table rase du discours normatif des spécialistes de littérature bretonne (dans lequel il est bien difficile de séparer ce qui relève du propos - lorsqu'on y accède - des détenteurs de la tradition chantée de ce qui relève de la construction du chercheur), et repartir de véritables enquêtes ethnolinguistiques rendant compte des catégorisations vernaculaires, terroir par terroir? 


\section{BIBLIOGRAPHIE}

AR FLOC'H Loeiz et FOUCHER Jean-Pierre, 1977, Le brasier des ancêtres. Poèmes populaires de la Bretagne, Paris, U.G.E., coll. 10-18.

EVANS E., 1977, « Some Celtic Forms in cant- », The Bulletin of Board of Celtic Studies, XXVII-2: 239-245.

GASTOUÉ A., 1924, Le cantique populaire en France. Ses sources, son histoire augmentés d'une bibliographie générale des anciens cantiques et noëls, Lyon, Editions Musicales Janin, Bibliothèque de l'art musical religieux.

GIRAUDON Daniel, 1985, Chansons populaires de Basse-Bretagne sur feuilles volantes, Morlaix, Skol Vreizh.

GOURVIL François, 1960, Th. Cl. H. Hersart de La Villemarqué et le Barzaz Breiz, Rennes.

GUYONVARC'H Ch.-J. et F. LE ROUX, 1986, Les druides, Rennes.

HAMELINE Jean-Yves, 1979, « Cantiques et complaintes. I. Domaine catholique », La religion populaire, Paris, CNRS : 379-381.

HAMELINE Jean-Yves, 1981, « Le cantique sur vaudeville à l'époque de Montfort et de Pellegrin », Ethnologie Française, tome 11, $\mathrm{n}^{\circ} 3$.

HERRIEU L. et M. DUHAMEL, 1911, Chansons populaires du pays de Vannes, Paris, Rouart, Lerolle et Cie.

KEMENER Yann-Fañch, 1996, Carnets de route, Morlaix, Skol Vreizh.

LAUNAY Denise, 1993, La musique religieuse en France du Concile de Trente à 1804, Paris, Publications de la Société Française de Musicologie, Klincksieck.

LAURENT Donatien, 1967, « La gwerz de Louis Le Ravallec », Revue des A.T.P., 15, n 1 : 19-79.

LAURENT Donatien, 1971, « La gwerz de Skolan et la légende de Merlin », Ethnologie française, 1, n ० 3-4 : 19-54.

LAURENT Donatien, 1989, Aux sources du Barzaz-Breiz. La mémoire d'un peuple, Douarnenez, ArMen.

LAURENT Donatien, 1988, « Histoire et poésie chantée : l'exemple de la Bretagne », Bretagne clés en mains, s. l., A.P.H.G. et Institut Culturel de Bretagne : 111-115.

LA VILLEMARQUÉ Hersart de, 1959 [1867], Barzaz-Breiz, Paris, Librairie Académique Perrin.

LE DIBERDER Yves, 1911-1912, [Critique de l'ouvrage de Duhamel et Herrieu, Chansons populaires du Pays de Vannes], Annales de Bretagne, tome 27 : 531-537.

LE ROUX Pierre, 1977 [1924], Atlas linguistique de la Basse Bretagne, Rennes, Plihon et Homnay, Paris, Champion, Brest, Editions Armoricaines et CDDEP.

LE MENN Gwenolé, s. d., Le chant populaire en Basse-Bretagne, Centre de Télé-Enseignement de l'Université de Haute-Bretagne, Civilisation bretonne, document $n^{\circ} 12$.

LUZEL François-Marie, 1971 [1868-1890], Chants et chansons populaires de la Basse-Bretagne, Paris, Maisonneuve et Larose, vol. 1. 
MARTIN Hervé, 1988, Le métier de prédicateur à la fin du Moyen âge, 1350-1520, Paris, Cerf.

PIRIOU Yann-Ber, 1989, «Les Bretons sous l'Ancien Régime d'après les gwerzioù de F.-M. Luzel », La Bretagne, une province à l'aube de la Révolution, Brest-Quimper, C.R.B.C.-S.A.F. : 233-247.

POPIN M.-D., 1987, « Le versus et son modèle », Revue de Musicologie, tome 73, n $1:$ 19-38.

QUELLIEN Narcisse, 1899, Chants et danses des Bretons, Paris, Maisonneuve et Leclerc.

RIVIÈRE Hervé, 1994, Les cantiques de pardons en Basse-Bretagne. Du rite au rythme, Thèse, Paris IV, Paris-Sorbonne.

TANGUY Bernard, 1977, Aux origines du nationalisme breton, Paris, coll. 10-18, tome 2.

ZUMTHOR Paul, 1987, «Les marques du chant. Le point de vue du philologue », Revue de Musicologie, tome 73-1:9-10.

\section{NOTES}

1. Certains cantiques, tel le Da Feiz hon tadoù kozh («A la foi de nos pères ») dont la fonction identitaire en fait un véritable hymne national, sont parfois chantés dans des circonstances totalement autres (banquets, assemblées, etc.). Distinguons cependant le contexte premier et les contextes dérivés.

2. Cf. Rivière 1994. Tous les termes bretons isolés seront transcrits dans la graphie dite « interdialectale » (par exemple, « kanenn » représente « kañnen », « cannen », etc.). Les citations d'auteurs gardent leur orthographe d'origine.

3. Le sigle KLT utilisé par les celtisants renvoie aux initiales bretonnes de Cornouaille, Léon et Trégor.

4. Partie occidentale du Vannetais.

5. Partie orientale du Vannetais. Cette seconde réalisation de l'opposition kanenn / sonenn est celle en vigueur dans la littérature vannetaise écrite.

6. Cf. également l'adoption de ce terme dans la musique médiévale, religieuse (versus, variété de tropes) ou profane (vers est le terme primitif de la canso des troubadours). Versus passera de «vers chanté » à " vers » tout court, laissant à son le soin de désigner plus spécifiquement le support musical (cf. Zumthor 1987 : 9-10) (cf. aussi Popin 1987 : 19-38).

7. Gwerz(enn) désigne par ailleurs le vers, la strophe ou le poème. Dans la langue des lettrés vannetais, gwerzenn désigne parfois tout texte rimé chanté, profane ou religieux. Cette acception a été généralisée dans les publications à vocation populaire au $\mathrm{xx}^{\mathrm{e}}$ siècle ( $c f$. notamment la revue Dihunamb, 1905-1944) (cf. aussi, en 1723, le dictionnaire vannetais de P. de Châlons : « Guerzeen, pl. guerzenneu, toute sorte d'Histoire ou Contes, Chansons »).

8. Cf. Laurent 1967.

9. " An dud 'lâra o foen ba'r ganenn. Ur gwerz (sic) a zo savet c'hoazh âr tud ha'n eus gwelet vil, ha'zo bet lazhet ha traou sort-sen. Goude 'mant bet lazhet 'vez savet ur ganenn âr o buhe [...].Ur werz a zo ur ganenn trist, ur ganenn poenius hag a lâra buhedigezh un den, peotramant buhedigezh zo bet, hag a gonta un dra trist [...]. Mes buhedigezh ataw » (entretien avec Monique Le Boulch, Inguiniel, 13 octobre 1989).

10. «Il était une fois... »

11. Entretien avec Yann-Fañch Kemener, Quimper, 21 juin 1990.

12. Cela dit, toute gwerz au contenu religieux n'est pas pour autant un chant d'Église. Pour accéder au rang de cantique, encore faut-il que l'autorité ecclésiale la reconnaisse comme tel ( nihil obstat ou imprimatur).

13. Dans leur utilisation en français, gwerz et son sont, selon les auteurs, tantôt masculins tantôt féminins. Leurs pluriels sont gwerzioù et sonioù. 
14. Dans la préface de sa célèbre anthologie intitulée Barzaz Breiz. La première édition de cette anthologie date de 1839, mais c'est la troisième, celle de 1867, qui servira de base aux rééditions ultérieures. L'ouvrage, admiré par George Sand, resta pendant près d'un demi-siècle une référence en matière d'édition de chants populaires.

15. A l'admiration vouée au Barzaz Breiz succéda une vague d'attaques, parfois très violentes, dénonçant les mystifications historiques, la falsification des textes recueillis - quand il ne fut pas dit que l'ouvrage était une invention pure et simple. Le détracteur le plus zélé du vivant de La Villemarqué fut sans doute Luzel, qui se lança, à son tour, dans la collecte et la publication de chants, avec une rigueur sans commune mesure avec l'œuvre littéraire de son prédécesseur. On trouvera la somme de griefs retenus contre La Villemarqué dans la thèse de François Gourvil (1960), et leur pondération dans une autre thèse, celle de Donatien Laurent (1989).

16. La contradiction n'est peut être qu'apparente. On peut en effet imaginer que les personnes interrogées ont répondu en fonction du "genre " dominant: là où la gwerz l'emporte, les informateurs ont donné « gwerz »; là où la son l'emporte, ils ont donné « son ».

17. «Outre son, on peut aussi bien avoir chanson ou Kanaouenn» (Giraudon $1985: 64$ ); « Kanaouen [...] est un terme purement générique [...]. Cependant kanaouen et sonn sont pris souvent l'un pour l'autre; et même, dans le dialecte de Tréguier, kanoen est employé généralement, sans aucun synonyme, dans le sens de sonn " (Quellien 1899 : 12).

18. A vrai dire, la lecture même des termes cantalon, cantlon, etc. sur les documents archéologiques, ainsi que le sens exact qui doit leur être attribué, posent problème ( $C f$. Evans 1977 : 239-245). Nous nous référons ici à la lecture de Ch.-J. Guyonvarc'h et de F. Le Roux (1986 : 372).

19. Notons la même autre acception de part et d'autre de la Manche : «vers ", "groupe de vers » ( « strophe ») - voire " poème » - dans leur seule dimension textuelle. Il est également intéressant de noter que kentel peut avoir, en moyen-breton, le sens de «temps» et qu'il rejoint donc l'acception moderne de « gwerz » dans « gwerso » (« gwerz zo »), « il y a longtemps ». (Cf. le français « cours »- du temps, et « leçon ».)

20. La «Chanson de fête des petits pâtres » a pour titre, en breton, « Kentel fest ar vugale » (Barzaz Breiz, p. 438). Le premier couplet du «Pardon de Saint-Fiacre» (Barzaz Breiz, p. 351) commence ainsi : "Tostait holl, tud iaouang, ha c'hui re goz ive, / Hag e klefot eur gentel [c'est nous qui soulignons] zo savet a neve [...]». Ce qui est traduit: "Approchez tous, jeunes gens, et vous vieillards aussi ; et vous entendrez un chant nouvellement composé ».

21. Cf. par exemple Donatien Laurent 1971 : 29. Relevons d'ailleurs une certaine contradiction : si La Villemarqué place kentel dans la catégorie des son, Donatien Laurent la place plutôt dans celle des gwerz.

22. Cf. les rectifications faites dans les Annales de Bretagne par Yves Le Diberder au sujet de la préface de Herrieu et Duhamel. Notamment : «Il est bien léger [...] de prétendre que les guerzes que l'on chante en Pays de Vannes sont généralement 'importées' (1911-1912 : 532).

23. Cf. les définitions de Laurent ou de Kemener mentionnées plus haut p. 61 (ou encore celle de Le Floc'h et Fouchez $1977: 17)$. Aucune ne précise si ces chants religieux peuvent être des cantiques officiels. Quellien, en évoquant ces clercs «qui transforment en cantique un vieux gwerz pieux » (1899: 14), montre par contre qu'il fait bien la part des choses entre chant d'Église et, si l'on peut dire, «chant religieux du domaine profane ».Kemener traduit le titre du premier chapitre de ses Carnets de route, "gwerzioù relijiel» (mot-à-mot «gwerz religieuses »), par « chansons d'inspiration religieuse » (1996: 87). Les pièces réunies sous cette rubrique sont assez hétérogènes (chants de colportage, chants de transmission proprement orale, etc.). Il est quand même surprenant d'y découvrir des chants de quête, notamment, dont la structure narrative est indigente. Sont-ils réellement appelés gwerz par les chanteurs? Le syntagme " gwerzioù relijiel » utilisé ici par Kemener serait-il approuvé par les chanteurs pour tous les chants du chapitre? 


\section{RÉSUMÉS}

Le classement tripartite des "genres " par les folkloristes et les spécialistes de littérature orale bretonne a été présenté, au moins au moment de son élaboration, comme fondé sur celui des chanteurs eux-mêmes. Mais la validité de cette tripartition - qui a tendance à saturer le discours savant - a-t-elle été vérifiée sur l'ensemble de la Bretagne bretonnante ? Sur un autre plan, à quoi attribuer les quelques zones de flou contenues dans les définitions? A une incohérence du discours autochtone de référence, ou à une mauvaise compréhension de ce dernier par les folkloristes? L'examen de la terminologie vernaculaire relative aux chants religieux permet d'entrer de plain-pied dans la problématique plus générale de la catégorisation poético-musicale bretonne. L'auteur en vient à militer pour que cette dernière soit à nouveau entreprise, mais cette fois-ci sur une base ethnolinguistique nettement plus affirmée.

The tripartite classification by folklore experts and those specialising in an orally based literary tradition in Brittany is generally present as be based on that of the singers themselves. However, has the validity of this tripartite approach, which has a tendency to dominate learned discourse on this topic, been fully checked vis à vis the whole of Breton speaking Brittany? From another perspective, to what might one attribute a distinct lack of clarity regarding some definitions? Can this be laid at the door of a fundamental incoherence in the native classification or simply a lack of understanding on the part of folklore experts? An assessment of vernacular terminology regarding religious chant brings us ultimately into the more general classification of Breton 'poetic-music'. The author suggests that this problem be restudied in a clearly ethnolinguistic perspective.

\section{AUTEUR}

\section{HERVÉ RIVIÈRE}

Hervé Rivière est docteur en musicologie. Chargé de recherche au Centre National de la Recherche Scientifique, il travaille au Département d'ethnomusicologie du Lacito (laboratoire de Langues et Civilisations à Tradition orale, UPR 3121 du CNRS, Paris). 